\title{
Entre scatologie et fantasmes sexuels, le cul et son imaginaire
}

Michel Biard et Pascal Dupuy

\section{(2) OpenEdition}

Journals

Édition électronique

URL : https://journals.openedition.org/ahrf/11660

DOI : $10.4000 /$ ahrf. 11660

ISSN : 1952-403X

Éditeur :

Armand Colin, Société des études robespierristes

Édition imprimée

Date de publication : 1 septembre 2010

Pagination : 3-11

ISBN : 978-2-200-92633-5

ISSN : 0003-4436

Référence électronique

Michel Biard et Pascal Dupuy, "Entre scatologie et fantasmes sexuels, le cul et son imaginaire », Annales historiques de la Révolution française [En ligne], 361 | juillet-septembre 2010, mis en ligne le 01 septembre 2013, consulté le 22 avril 2022. URL : http://journals.openedition.org/ahrf/11660 ; DOI : https://doi.org/10.4000/ahrf.11660

Ce document a été généré automatiquement le 22 avril 2022.

Tous droits réservés 


\title{
Entre scatologie et fantasmes sexuels, le cul et son imaginaire
}

\author{
Michel Biard et Pascal Dupuy
}

\section{NOTE DE L'ÉDITEUR}

Les images qui accompagnent les articles ont été rassemblées dans deux cahiers distincts, en documents annexes. La formule « cahier couleur » placée à la suite d'un numéro de renvoi d'une illustration indique que celle-ci est placée dans le cahier couleur. Si le numéro de renvoi n'est pas suivi de cette formule, l'image se trouve dans le cahier noir et blanc.

1 En vertu d'un long processus de « civilisation des mœurs »", ainsi que des convenances mondaines qu'il convient de respecter en "société", nul ne peut se hasarder au XVIII ${ }^{\mathrm{e}}$ siècle à montrer en public son derrière, « cette partie de l'homme qui comprend les fesses \& le fondement " ${ }^{2}$, ni même se laisser aller à lâcher un « vent ", a fortiori l'une de ces $"$ vesses $\|^{3}$ dont la puissance de l'odeur est inversement proportionnelle à celle $\mathrm{du}$ son et ravale au rang de faible protection pour la pudeur tant la superbe culotte de l'aristocrate que les guenilles de l'indigent ${ }^{4}$. De la même façon, rares sont ceux qui osent faire état publiquement des plaisirs qu'ils recherchent dans une partie du corps dont morale et religion réprouvent tout usage sexuel, d'autant qu'il s'agit là de pratiques jugées odieuses et contraires à l'idée même d'un «devoir conjugal » avant tout destiné à la procréation. Faut-il le rappeler ? Dans la France des Lumières existent de nombreuses façons d'appliquer la peine capitale et parmi elles certaines sont tenues pour être « cruelles », comme l'écartèlement, la roue et le bûcher. Ce dernier supplice a longtemps servi à anéantir ceux et celles qui étaient accusés d'hérésie ou de sorcellerie, mais sans doute a-t-on trop souvent oublié que les flammes supposées purificatrices servaient aussi à envoyer en « enfer » les individus reconnus coupables de zoophilie et les «bougres », qu'ils soient « bougrants », « bougrés » ou les deux alternativement. En effet, pour ceux qui préféraient être "culistes " plutôt que " conistes ", et surtout qui choisissaient de «fourgonner les anus $\|^{5}$ masculins, la justice se veut alors exemplaire 
et ce n'est qu'avec le Code pénal de 1791 que le "crime de sodomie " disparaît. Pourtant, si l'intime doit ainsi être pudiquement voilé, tant la chair que les plaisirs qu'elle peut exciter, le mot «cul » marque aussi de sa présence tout un vocabulaire et un imaginaire qui tiennent à la jouissance du monde à l'envers cher au Carnaval, lorsque le cul se retrouve par-dessus tête ${ }^{6}$, mais aussi à toute une prose qui aime à employer des mots et expressions appartenant au registre du « bas langage $»^{7}$. Nombre d'expressions ordonnées autour du mot «cul», et employées dans ce XVIII ${ }^{\mathrm{e}}$ siècle où l'art de la conversation voisine avec des formes de langage populaires, ont traversé les décennies jusqu'à notre actuel langage. Si nous ne disons plus guère " foutre la pelle au cul », «se tenir par le cul comme des hannetons » ou encore «remuer de cul et de tête", qui de nous n'a entendu ou utilisé des expressions comme "lécher le cul», "mettre des coups de pied au cul», voire "péter plus haut que le cul»? ? De tout cela, la Révolution a tôt fait de s'emparer dès lors que le grotesque peut servir à ridiculiser l'adversaire, que le mot «cul " peut rendre un article de journal ou un pamphlet plus piquants et donc d'une diffusion plus grande, qu'une bonne fessée «le cul au vent » attire toujours les rires (et le voyeurisme) autant que les coups de bâton chers aux théâtres de foire, enfin que le ci-devant "crime de sodomie » permet aisément de disqualifier un homme à abattre.

2 Révolution laissant libre cours à des propos et/ou des actes «libertins ", voire numéro des $A H R F$ «libertin»? Il ne s'agit naturellement en rien de s'égarer. L'épithète de libertin a traversé les $\mathrm{XVII}^{\mathrm{e}}$ et $\mathrm{XVIII}^{\mathrm{e}}$ siècles. Au XVII ${ }^{\mathrm{e}}$ siècle, les libertins étaient présents et actifs, mais souvent peu visibles. Pour plusieurs d'entre eux, le libertinage consistait en une philosophie irreligieuse marquée, tout particulièrement, par un refus des dogmes chrétiens. Pendant la Fronde, quelques jeunes nobles proclament des discours blasphématoires dont les chansons nous permettent d'envisager les repas où ces couplets étaient entonnés, puis repris en chœur :

«Qu'une colombe à tire d'aile

Ait obombré une Pucelle

Je ne crois rien en tout cela.

On en dit autant de Phrygie,

Et le beau cigne de Léda

Vaut bien le pigeon de Marie "

3 À côté de ces libertins de Cour, portant haut leur incrédulité, d'autres avaient pour principal souci de dissimuler leur détachement religieux en une sorte de «libertinage critique $»^{10}$. Si ces derniers affectaient par prudence de ne pas s'en prendre ouvertement à la religion chrétienne, ils relevaient les « ruses et les supercheries » des fondateurs de religion, ce qui conduisait inévitablement leur lecteur à porter un regard critique sur l'enseignement du Christ. Toutefois, ces hommes (La Mothe le Vayer, Gabriel Naudé, etc.) remettaient moins en cause l'ordre religieux et politique établi, qu'ils ne s'attaquaient aux préjugés, aux miracles et aux superstitions contraires à la raison. Si les libertins du XVII e siècle se montraient rarement blasphématoires, ils n'en ont pas moins ouvert la voie à l'affirmation à venir de l'athéisme et du déisme. En outre, en passant par le libertinage épicurien d'un Saint-Évremond, de sa soif de volupté et de jouissance, le libertinage a valorisé la recherche du plaisir comme un bien, attitude qui s'épanouira, avec un glissement sémantique, dans la figure du «libertin" au xvIII ${ }^{\mathrm{e}}$ siècle. Le libertinage quitte alors la sphère du rapport métaphysique au divin dans laquelle il était enfermé au siècle précédent pour envahir le domaine de l'esthétique, des belles lettres et du discours mondain. Le libertin devient 
alors un débauché, adepte du plaisir sensuel et du dévergondage ${ }^{11}$. Bien plus que Valmont dans Les Liaisons dangereuses, le marquis de Sade n'en deviendra-t-il pas, jusqu'à la radicalité, le modèle ultime? Son œuvre est marquée par une véritable "obsession anale", pour reprendre les mots de Michel Delon, qui constate ailleurs qu'elle «[...] se comprend dans le vaste mouvement de renoncement aux règles du bien-dire, qui accompagne la revendication de l'originalité et de l'authenticité. La volonté de "tout dire" de l'auteur des Cent Vingt Journées de Sodome rejoint paradoxalement la nudité autobiographique de Rousseau, auquel il s'oppose fréquemment $[. ..] »^{12}$. «Tout dire », c'est, entre autres, décrire jusqu'à saturation les actes sexuels, avec un nombre de partenaires variable - mais des pratiques pas si variées - et surtout avec ce qui met en scène tout cul à "foutre » comme l'explique Dolmancé à Eugénie : "On appelle bougres ceux qui se livrent à cette passion; or, quand on fait tant que d'être bougre, Eugénie, il faut l'être tout à fait. Foutre des femmes en cul n'est l'être qu'à moitié : c'est dans l'homme que la nature veut que l'homme se serve de cette fantaisie; et c'est spécialement pour l'homme qu'elle nous en a donné le goût ${ }^{13}$. Quant à ce qu'il baptise le " prétendu crime » de sodomie, Sade n'oublie pas de mentionner implicitement le Code pénal de 1791 : « Est-il possible d'être assez barbare pour oser condamner à mort un malheureux individu dont tout le crime est de ne pas avoir les mêmes goûts que vous? [...] Consolez-vous, citoyens, de telles absurdités n'arrivent plus : la sagesse de vos législateurs vous en répond ${ }^{14}$. Le divin marquis accompagne un temps la Révolution, mais celle-ci, en imaginant un monde meilleur, fondé sur le bonheur et bannissant la souffrance inutile, lui est aussi insupportable. Très vite, à ses yeux, « la guillotine qui veut dénouer le corps à corps du bourreau et de la victime, réduire les souffrances du condamné, est l'antithèse de toutes les machines dont il rêve avec effroi et délectation $»^{15}$.

4 Notre projet pour ce numéro des AHRF a été d'explorer autant les mutations esthétiques que les profondes continuités de cette partie du corps humain que l'on désigne poliment sous le nom de "derrière", mais que les caricatures du temps dévoilent avec jubilation ou qu'Hébert et son Père Duchesne emploient fréquemment, ici pour railler Marie-Antoinette qui «tortille du cul » pour mieux séduire et tromper ses interlocuteurs, là pour s'en prendre aux aristocrates qu'il faut chasser « à coup de pied au cul $»^{16}$ comme de vulgaires manants. La «mise à nu du cul», outre qu'elle peut ici préluder au châtiment, se veut toujours humiliante car elle «[...] impose une révélation qui abolit la frontière du privé » (Stéphanie Génand). La Révolution abolit aussi les châtiments corporels qui accompagnaient les condamnations, et même la peine capitale ne met plus à l'ouvrage que la seule guillotine ${ }^{17}$ ? Qu'à cela ne tienne, le cul peut servir de défouloir et le fustiger suffit à remplacer la ci-devant flétrissure de la chair par la justice. Le cul est donc en bonne place lorsque, au cours de la Révolution, on veut expulser l'ennemi en lui foutant «la pelle au cul» ou le ridiculiser en l'obligeant à se défroquer ou encore, comme dans les images, en le représentant dans sa plus stricte intimité en train de s'adonner à des besoins naturels, position propice au détournement scatologique et aux fantasmes à propos des excréments. De son côté, dans cet univers encore sensible aux jeux et aux règles carnavalesques, le pet est utilisé comme une autre fonction dévalorisante, même s'il pouvait prendre les formes, au XVIII ${ }^{\mathrm{e}}$ siècle, chez Rousseau par exemple dans Les Confessions, d'une sorte de trait d'esprit macabre : « [Mme de Vercellis] ne garda le lit que les deux derniers jours, et ne cessa de s'entretenir paisiblement avec tout le monde. Enfin, ne parlant plus, et déjà dans les combats de l'agonie, elle fit un gros pet. Bon, dit-elle en se retournant, femme qui pette 
n'est pas morte. Ce furent les derniers mots qu'elle prononça ${ }^{18}$. Le XvIII ${ }^{\mathrm{e}}$ siècle et la Révolution observent le pet, comme nous l'indique Philippe Bourdin, dans des manuels et autres Éloge du pet, associant aux relents de "réjouissances populaires» des développements sur l'origine du mot, ses vertus médicinales ou sa musicalité.

Ces textes et images satiriques découvrent ce que généralement l'on souhaite cacher en une sorte de révélateur de l'intimité de l'âme et du caractère. L'adversaire est alors évoqué à travers ses coupables penchants, les plus intimes étant les plus révélateurs. Cambacérès, comme l'explique Emmanuel Fureix, est ainsi assimilé dans les images à un " sodomite» afin de le dépourvoir de toute crédibilité publique (« dans les caricatures contre Cambacérès, les fesses rebondies, les regards lubriques et autres jeux de mots grivois ne doivent pas tromper: derrière les cancans et la dérision se cache un dispositif politique efficace, capable de mettre hors-jeu un acteur incommode "). Pendant la Révolution, l'aristocrate avait déjà revêtu ce costume, embarrassant pour la morale, d'adepte de plaisirs hédonistes que l'on dénonce comme anti-naturels et antipatriotiques. L'homosexualité masculine se charge alors d'une fonction politique négative, contre-nature, que Thierry Pastorello assimile également à une volonté de régénération des mœurs, l'Ancien Régime étant identifié à la corruption et à la débauche. Cette évocation de la débauche était déjà présente dans la période prérévolutionnaire comme l'ont déjà montré de nombreux chercheurs et certains auteurs ont même vu dans sa recrudescence, dont témoigne la littérature clandestine de la fin $\mathrm{du}$ siècle, des indications claires du désarroi des autorités ${ }^{19}$ et d'une perte des repères que la Révolution accentua. Comme on le sait, le nombre d'écrits politicopornographiques s'accroît de 1774 à 1788 , puis se multiplie à partir de $1789^{20}$. Dans cette littérature, le cul est omniprésent. Ainsi, le goût pour les artifices de la mode, et tout particulièrement celui des chapeaux à plumes, est utilisé régulièrement comme un indicateur de perversions coupables. L'Almanach des honnêtes femmes pour l'année 1790 décrit ainsi un "petit juif [qui] déshabillait sa maîtresse, lui enfonçait des plumes de paon dans le derrière, et la faisait promener dans la chambre à quatre pattes; il lui passait ensuite la main sur la croupe, et s'écriait: "Oh! le bel oiseau !" Lui-même se prêtait à cette métamorphose, et il finissait, après quelques moments d'extase, par prendre la place des plumes ${ }^{21}$. Comme l'indique Chantal Thomas, on trouve également un récit similaire où cette fois le prêtre remplace le juif sans toutefois altérer le message politique et réprobateur du texte : «Il tire alors de sa poche une belle plume de queue de paon et déboutonne sa culotte, puis il se couche le cul en l'air sur mon lit, il me dit de lui insérer le bout de cette plume dans le fondement, ce que je fis; ensuite de lui caresser le cul avec la main en prononçant ces paroles: "Ah! le beau paon!" ${ }^{22}$. Dans cette littérature pornographique, le cul de la reine est un objet de désirs et de reproches souvent mis en scène et c'est d'ailleurs la Vie privée, libertine, et scandaleuse de Marie-Antoinette qui, en 1790-1791, vient en tête des pamphlets saisis par la police. Comme l'a montré Lynn Hunt $^{23}$, le corps de la reine devient à la fois un objet témoin de la dégradation de la monarchie, mais aussi, dans la mesure où il semble accessible à tout le monde, la figure d'accession perverse à la notion de souveraineté. Dans ces images et dans ces textes, le cul de la reine est une donnée de la licence et de la perversion de celle qui fut considérée comme la Messaline française. Ainsi, dans les célèbres Amours de Charlot et Toinette de 1779 (soit le comte d'Artois et MarieAntoinette), l'auteur parle sans détour du prince qui «baise ses beaux bras, son jolie petit con, et tantôt une fesse et tantôt un téton : Il claque doucement sa fesse rebondie, cuisse, ventre, nombril, le centre de tout bien $»^{24}$. Dans un autre pamphlet, publié en 
1789, sous la forme d'un "opéra proverbe, composé par un Garde-du-Corps », on aperçoit Artois découvrant «les deux fesses blanches comme de la neige » de la reine. Ce « cul blanc » est comme une invitation à la fessée, même si dans la suite de l'opéra ce n'est pas à ce caprice que s'adonne le frère du roi lequel, aidé par l'une des confidentes de Marie-Antoinette, « introduit un doigt léger sur le portique du Temple dans lequel le comte s'introduit par une voie détournée $»^{25}$.

6 La fessée, même si elle n'est pas fréquemment associée à la reine, est cependant, comme nous le révèle Annie Duprat, un élément moteur classique du rire et de la satire, utilisée par les images de manière à la fois transgressive, blasphématoire, anti-cléricale mais peut-être avant tout et fondamentalement patriotique et propitiatoire. La fessée et la flagellation procèdent d'une longue tradition érotique qui semble particulièrement développée en Grande-Bretagne. Les pamphlets français qui les mentionnent à la fin du XVIII ${ }^{\mathrm{e}}$ siècle en font même une spécialité anglaise. En effet, les années 1770 ont connu en Grande-Bretagne l'apparition de nombreux ouvrages clandestins évoquant ces pratiques, supposées, tout particulièrement, se produire entre femmes. Elles révèlent des comportements dont certaines tenancières de maisons closes s'étaient faites les spécialistes, comme Theresa Berkley dont l'établissement était situé sur Charlotte Street à Londres et qui republia à ses frais, en 1793, un ouvrage classique et fondamental sur le sujet ${ }^{26}$. Comme l'indique un historien britannique ${ }^{27}$, il est également intéressant de constater que ces pratiques étaient directement associées au monde de l'aristocratie anglaise. Dès le XVII ${ }^{\mathrm{e}}$ siècle, des pamphlets accusaient les aristocrates d'y avoir pris goût dans des écoles privées où la flagellation était une forme de punition traditionnelle. Mais, on constate que, dans le même temps où se développait cette littérature autour de la flagellation, apparaissent de nombreuses accusations pour sodomie. La croupe et les fesses prennent une importance croissante dans les pratiques érotiques britanniques que le célèbre ouvrage pornographique Fanny Hill a mis en valeur :

«Elle présentait une pleine vue d'arrière de sa personne nue jusqu'à la ceinture. Son postérieur charnu, lisse et proéminent formait une double et luxuriante nappe de neige animée qui remplissait glorieusement l'œil et suivant la pente de ses blanches collines dans l'étroite vallée qui les séparait, s'arrêtait et s'absorbait dans la cavité inférieure... $»^{28}$.

7 Si l'on suit ce développement, les fesses, comme objet du désir, prennent une place grandissante à la fin du $\mathrm{XVIII}^{\mathrm{e}}$ siècle, ce qui explique également leur présence envahissante dans les caricatures du temps. À cet égard, le cul dans les gravures satiriques britanniques se révèle à la fois un objet érotique, mais bien sûr et plus profondément, un moteur de la transgression au travers de mécanismes traditionnels de la politisation de masse. Wolfgang Cillessen et Rolf Reichardt ont ainsi repéré quelques thèmes scatologiques qui parcourent l'imagerie satirique européenne de la Réforme au milieu du XIX ${ }^{\mathrm{e}}$ siècle. Ces motifs ont en commun d'associer la dégradation de l'image de l'adversaire et le message politique. Sous couvert du grotesque, s'insinue une propagande qui va au plus profond des désirs et des refoulements dans une société travaillée à la fois par l'imposition des bonnes mœurs policées et les frustrations les plus enfouies. La satire par le cul dans la caricature française prend l'allure d'un dénigrement du corps avili et malade qui est un obstacle à la régénération de la société. L'aristocrate en est la cible désignée, tandis que du côté anglais le cul prend plutôt des allures comiques et grossières caractérisant à l'opposé le révolutionnaire français, au propre comme au figuré, sans culotte. 
8 Si le sujet de tous ces articles et l'objet général de ce numéro des AHRF pourront sans doute prêter à sourire, il nous semble pourtant important pour ceux et celles qui veulent apprécier à la fois les nouvelles formes de la contestation politique mais aussi ses continuités, et ainsi appréhender, de manière certes triviale et souvent grossière, l'imaginaire d'un monde en mutation accélérée. Rappelons en effet que ce temps où le cul fut un moment par-dessus tête, loin d'avoir été celui du désordre que se sont plu à condamner nombre d'hommes de plume hostiles à la Révolution, dès l'événement et parfois hélas jusqu'à nos jours, a fondamentalement donné naissance à un nouvel ordre. Plus tolérant vis-à-vis du cul et de ses multiples usages ? Voire...

\section{NOTES}

1. Norbert ÉLIAS, La civilisation des mœurs, Paris, Calmann-Lévy, 1973.

2. Dictionnaire de l'Académie, édition de 1694.

3. Le dictionnaire cité ci-dessus décrit ainsi le phénomène : «Ventosité qui sort par le derrière sans faire bruit ".

4. Sur ce que Pierre Thomas Nicolas Hurtaut nomme en 1751 « l'art de péter », nous renvoyons à l'article de Philippe BOURDIN dans le présent numéro des AHRF ainsi qu'à l'introduction d'Antoine DE BAECQUE à la réédition récente du petit livre de HURTAUT (L'art de péter. Essai théori-physique et méthodique à l'usage des personnes constipées, des personnes graves et austères, des dames mélancoliques et de tous ceux qui restent esclaves du préjugé, Paris, Payot, 2006).

5. Voir dans le présent numéro l'article de Thierry PASTORELLO.

6. Voir dans le présent numéro l'article de Michel BIARD et Jacques GUILHAUMOU.

7. Dictionnaire du bas-langage, ou des manières de parler usitées parmi le peuple [...], Paris, d'Hautel et Schœll, 1808.

8. Sur ces expressions liées au mot «cul », voir Michel BIARD, Parlez-vous sans-culotte ? Dictionnaire du Père Duchesne 1790-1794, Paris, Tallandier, 2009.

9. Cité dans Antoine ADAM, Les libertins au XVII siècle, Paris, Buchet/Chastel, 1986, p. 77.

10. Ibid.

11. Et selon la célèbre formule de Crébillon fils, un homme «[...] qui se sert de l'amour pour assurer le triomphe de sa fantaisie au détriment de sa compagne, qui érige l'inconstance en principe et qui, recherchant uniquement le plaisir des ses sens et la satisfaction de sa propre vanité, n'accorde rien au sentiment dans l'entreprise de la conquête amoureuse " (Lettres de la marquise de M... au comte de R..., 1734).

12. Michel DELON, Introduction des Euvres de SADE, Paris, Gallimard, collection La Pléiade, 2000, p. LVII.

13. Marquis DE SADE, La Philosophie dans le boudoir, troisième dialogue. Immédiatement après cette tirade de Dolmancé, qui s'apprête à faire découvrir à sa jeune élève la "jouissance sodomite ", Madame de Saint-Ange s'empresse d'en tirer une conclusion: "Savez-vous Dolmancé, qu'au moyen de ce système, vous allez jusqu'à prouver que l'extinction totale de la race humaine ne serait qu'un service rendu à la nature ? »... Quant à la réponse de Dolmancé-Sade, elle se passe de commentaire : «Qui en doute, madame?» 
14. Ibid., citation extraite du fameux passage inséré dans le cinquième dialogue sous le titre « Français, encore un effort si vous voulez être républicains ».

15. Michel DELON, Introduction des Euvres de SADE, op. cit., p. LVII.

16. Voir, dans le présent numéro, l'article de Michel BIARD et Jacques GUILHAUMOU.

17. Au moins en droit, car il va de soi que nous n'oublions pas l'existence d'autres formes de mise à mort, notamment les pelotons d'exécution lors de certaines répressions de grande ampleur.

18. Euvres complètes de RousSEAU, Les Confessions, Paris, Armand-Aubrée, 1832, Volume II, Tome I, Livre II, p. 103.

19. Jeffrey MERRICK, "Sexual Politics and Public Order in Late Eighteenth-Century France ", Journal of the History of Sexuality, $\mathrm{n}^{\circ} 1,1990$, p. 68-84.

20. Voir Lynn HUNT, «Pornography and the French Revolution », in Lynn HUNT, The invention of Pornography. Obscenity and the origins of Modernity, 1500-1800, New York, Zone Books, 1993, p. 307.

21. Chantal thомаs, La reine scélérate. Marie-Antoinette dans les pamphlets, Paris, Seuil, 1989, p. 92-93.

22. Le Catéchisme libertin, 1791, p. 26.

23. Voir Lynn HUNT, « Pornography and the French Revolution », op. cit.

24. Les amours de Charlot et Antoinette, Pièce dérobée à V[ersailles], 1779. Voir Chantal THOMAS, La reine scélérate [...], op. cit., p. 171.

25. L'autrichienne en goguettes ou l'orgie royale. 1789. Voir Chantal THOMAs, La reine scélérate [...], op. cit., p. 195-196.

26. The Exhibition of Female Flagellants, Londres, 1793.

27. A. D. HARVEY, Sex in Georgian England, Londres, Phoenix Press, 1993, p. 36.

28. L'ouvrage de John CLELAND, Fanny Hill fut publié sous le titre Memoirs of a woman of Pleasure en 1749, puis un an plus tard sous le titre de Memoirs of Fanny Hill. Il fut interdit par les autorités. La traduction est tirée de Mémoires de Fanny Hill, femme de plaisir, Paris, Bibliothèque des curieux, 1910 (introduction par Guillaume Apollinaire), p. 232. 